(C) 2017 IEEE. Personal use of this material is permitted. Permission from IEEE must be obtained for all other uses, in any current or future media, including reprinting/republishing this material for advertising or promotional purposes, creating new collective works, for resale or redistribution to servers or lists, or reuse of any copyrighted component of this work in other works. 


\title{
IMPROVED TRANSMISSION AND THERMAL EMISSION IN MACROPOROUS SILICON PHOTONIC CRYSTALS WITH 700 nm PITCH
}

\author{
D. CARDADOR, D. VEGA, D.SEGURA AND A. RODRÍGUEZ \\ Micro i Nanotecnologies, Departament d'Enginyeria Electrònica, Universitat Politècnica de Catalunya, C/Jordi \\ Girona, 31, 08031, Barcelona, Spain. - E-mail: david.cardador@upc.edu. Tel: +34 934016766.
}

\begin{abstract}
Photonic Crystals (PCs) are becoming increasingly attractive for both research and market applications. Their optical properties, obtained by creating periodical structures in a material with a chosen refractive index, are very interesting for a wide range of application areas, such as optical communications or sensing. The introduction of defects that break the periodicity in the structure, confers the PC some interesting functionalities. An example is the fabrication of large defects that work as waveguides. However, planar or point cavities can be fabricated in order to couple light from outside the crystal. These kind of cavities create resonant states within the photonic bandgap at specific frequencies or modes what is used for developing optical resonators, thermal emitters, tunable filters, etc. [1].
\end{abstract}

It is well known that the material's structure -and, in concrete, the shape of the defect also- influences on the optical response of the photonic crystal. Several of articles have dealt with this issue in 1D and 2D PCs -i.e. [2], [3]. However, the influence of the defects morphology in the optical response of 3D photonic crystals have not been so deeply analyzed perhaps, because of the high dependence of the method used to fabricate the PC [4]. A number of different techniques have been proposed to incorporate defect structures within the PCs in woodpiles, synthetic opals or macroporous silicon, among others.

In this paper we focus on macroporous silicon (mp-Si), which is a versatile material that can be successfully fabricated through the so-called electrochemical etching (EE). With this technique it is possible to fabricate pores with different depth profiles -such as sinusoidal or straight- with a planar defect inside the crystal structure-see Fig. 1. Previous studies reported mp-Si structures with a cavity in the middle of the PC that had a resonant mode around the $7 \mu \mathrm{m}$ [5]. However, they worked with a pitch of $2 \mu \mathrm{m}$ and a vertical periodicity of about $2.5 \mu \mathrm{m}$. This vertical periodicity is limited to the lattice parameter: when approaching to the pitch value, the vertical periodicity is more difficult to achieve and the profile is harder to control by EE. Although some other studies reported modulated structures in $700 \mathrm{~nm}$ [6], they did not inserted a cavity in their structures. In the present study, the samples used to fabricate the macroporous silicon PCs had a pitch of $700 \mathrm{~nm}$. That allowed us to work with an initial vertical periodicity of about 1.1-1.2 $\mu \mathrm{m}$ what arouse a bandgap around [5-7] $\mu \mathrm{m}$. As depicted in Fig. 1, a planar defect was introduced halfway the total pore depth by suppressing one of the modulations and leaving a constant diameter section. The length of the cavity was $2.3 \mu \mathrm{m}$ and its width took the value of $0.23 \mu \mathrm{m}$. This cavity had a resonance frequency near $6.5 \mu \mathrm{m}$, as depicted in Fig. 2 .

In order to reduce the bandgap wavelength, and thus the position of the peak, the vertical modulation of the pore and the length of the cavity were shortened. In concrete, the periodicity was set to the lattice constant value $(\sim 700 \mathrm{~nm})$, the defect's length to $1.6 \mu \mathrm{m}$ and its width took the same value as before, $0.23 \mu \mathrm{m}$. Due to that modifications, the bandgap moved to the range of [4-5] $\mu \mathrm{m}$ and the resonance peak raised at $4.6 \mu \mathrm{m}$, where the $\mathrm{CO}$ has its absorption lines - see Fig. 2.

By removing around $150 \mu \mathrm{m}$ of bulk silicon of the samples, we were able to increase the transmission percentage from values around $4 \%-6 \%$ to $25 \%-30 \%$. This improves the features of the peak what, as a consequence, should improve the sensivity of the final gas sensor device.

In this paper we also present the thermal response of the two analyzed samples in order to see if they can be employed as a thermal emitter. Both samples were heated at a room temperature of $400{ }^{\circ} \mathrm{C}$. Comparing Fig. 2 and Fig.3, we observe that it exists a displacement between the transmission and absorption peaks that are attributable to the variations in the shape of the pores given by the expansion of the silicon due to the difference of temperatures.

Further studies have to be done to place the base of the peak as close as possible to the $0 \%$ of emission in order to have a real selective emitter.

This work is a starting point for gas detection devices using macroporous technology in the mid-infrared, which includes ammonia, formaldehyde, carbon monoxide or nitrus oxide, among others.

\section{References}

[1] P. V. Braun, S. A. Rinne, and F. García-Santamaría, "Introducing Defects in 3D Photonic Crystals: State of the Art," Adv. Mater., vol. 18, no. 20, pp. 2665-2678, Oct. 2006.

[2] I. Alvarado-Rodriguez, "Fabrication of twodimensional photonic crystal single-defect cavities and their characterization by elastic scattering," A Diss. Dr. Philos. Electr. ..., 2003.

[3] M. Mohebbi, "Refractive index sensing of gases based on a one-dimensional photonic crystal nanocavity," $J$. Sensors Sens. Syst., 2015.

[4] E. Nelson, "Three-dimensional photonic crystal optoelectronics," 2011.

[5] G. Mertens, R. B. Wehrspohn, H.-S. Kitzerow, S. Matthias, C. Jamois, and U. Gösele, "Tunable defect mode in a three-dimensional photonic crystal," Appl. Phys. Lett., vol. 87, no. 24, p. 241108, Dec. 2005.

[6] A. Langner, "Fabrication and characterization of macroporous silicon," Doktorarbeit, Martin-LutherUniversität, 2008. 


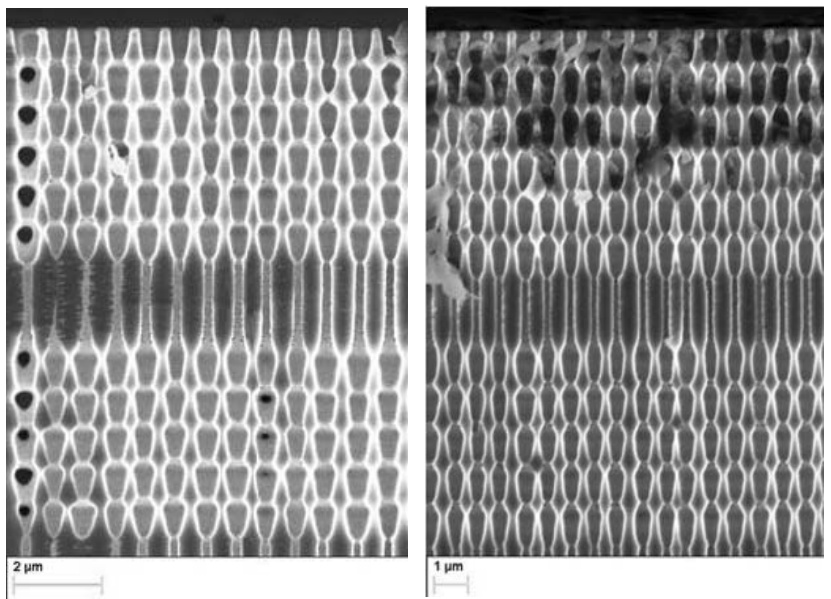

Fig. 1. Cross sectional view of two 3-D PCs fabricated by EE over a litography of $0.7 \mu \mathrm{m}$ pitch. The inclusion of a defect in the PC lattice enables a transmitted mode in $4.6 \mu \mathrm{m}$ (left) and $6.4 \mu \mathrm{m}$ (right).

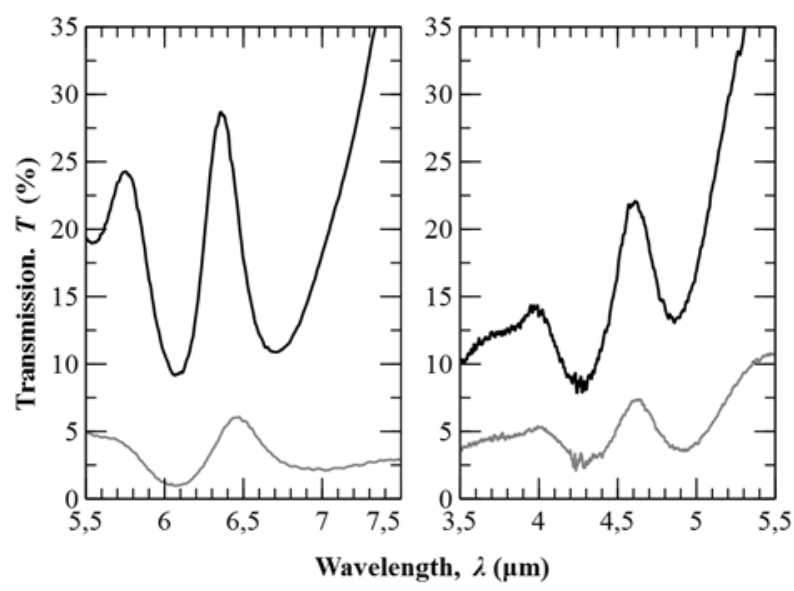

Fig. 2. Transmittance of the samples before (grey) and after (black) the TMAH attack for removing part of the bulk silicon of the wafer. The different wavelength range are achieved by controlling the vertically periodicity. Left image corresponds to the image plotted in Fig.1(a) and the right image to the Fig.1(b).

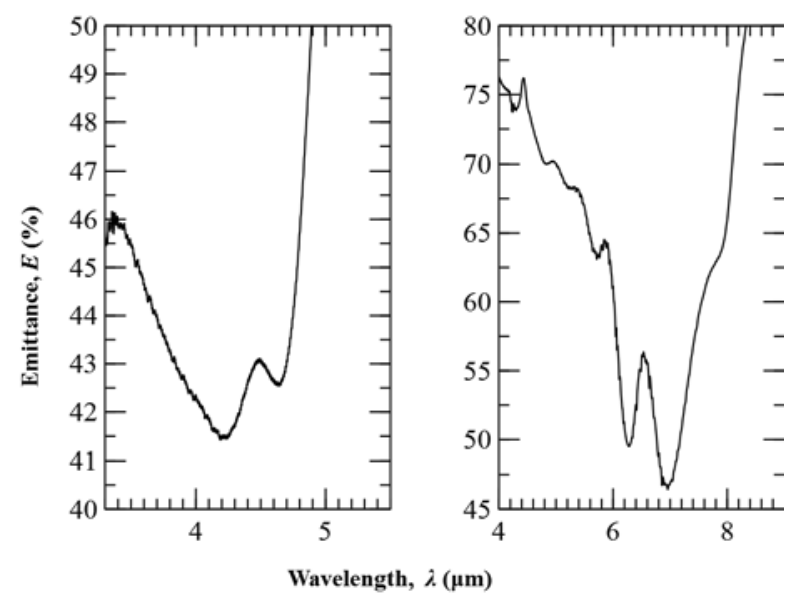

Fig. 3. Experimental emisison spectra for the samples depicted in Fig. 1 (they are conveniently depicted. E.g.: left image in Fig. 1 corresponds to the left image in Fig.3). Both samples were heated at $400{ }^{\circ} \mathrm{C}$. 